\title{
The Cosmic-Ray Contribution to Galactic Abundances of the Light Elements: Interpretation of GCR LiBeB Abundance Measurements from ACE/CRIS
}

\author{
N. E. Yanasak*, G. A. de Nolfo ${ }^{\dagger}$, W. R. Binns**, E. R. Christian ${ }^{\dagger}$, A. C. Cummings*, \\ A. J. Davis*, J. S. George*, P. L. Hink**, M. H. Israel ${ }^{* *}$, R. A. Leske*, M. Lijowski**, \\ R. A. Mewaldt*, E. C. Stone*, T. T. von Rosenvinge ${ }^{\dagger}$ and M. E. Wiedenbeck ${ }^{\ddagger}$ \\ ${ }^{*}$ Space Radiation Laboratory, California Institute of Technology, Pasadena, CA 91125 USA \\ ${ }^{\dagger}$ Laboratory for High Energy Astrophysics, NASA/Goddard Space Flight Center, Greenbelt, MD 20771 USA \\ ${ }^{* *}$ Dept. of Physics and McDonnell Center for the Space Sciences, Washington University, St. Louis, MO 63130 \\ USA \\ ${ }^{\ddagger}$ Jet Propulsion Laboratory, California Institute of Technology, Pasadena, CA 91109 USA
}

\begin{abstract}
Inelastic collisions between the galactic cosmic rays (GCRs) and the interstellar medium (ISM) are responsible for producing essentially all of the light elements $\mathrm{Li}, \mathrm{Be}$, and $\mathrm{B}(\mathrm{LiBeB})$ observed in the cosmic rays. Previous calculations (e.g., [1]) have shown that GCR fragmentation can explain the bulk of the existing $\mathrm{LiBeB}$ abundance in the present day Galaxy. However, elemental abundances of $\mathrm{LiBeB}$ in old halo stars indicate inconsistencies with this explanation. We have used a simple leaky-box model to predict the cosmic-ray elemental and isotopic abundances of $\mathrm{LiBeB}$ in the present epoch. We conducted a survey of recent scientific literature on fragmentation cross sections and have calculated the amount of uncertainty they introduce into our model. The predicted particle intensities of this model were compared with high energy $\left(E_{\mathrm{ISM}}=200-500 \mathrm{MeV} / \mathrm{nucleon}\right)$ cosmic-ray data from the Cosmic Ray Isotope Spectrometer (CRIS), which indicates fairly good agreement with absolute fluxes for $\mathrm{Z} \geq 5$ and relative isotopic abundances for all $\mathrm{LiBeB}$ species.
\end{abstract}

\section{INTRODUCTION}

It has been generally accepted for almost 30 years that production of the bulk of $\mathrm{Li}, \mathrm{Be}$, and $\mathrm{B}(\mathrm{LiBeB})$ in the present Galaxy can be attributed to inelastic collisions between cosmic rays and the interstellar medium (ISM). Specifically, large abundances of $\mathrm{LiBeB}$ arise from fragmentation of $\mathrm{C}, \mathrm{N}$, and $\mathrm{O}(\mathrm{CNO})$ in the ISM by cosmic ray $\mathrm{H}$ and $\mathrm{He}$, fragmentation of cosmic-ray $\mathrm{CNO}$ species by the $\mathrm{H}$ and $\mathrm{He}$ in the ISM, and $\alpha-\alpha$ fusion in collisions between cosmic-ray and ISM nuclei [2]. Many previous studies have shown that these mechanisms can generally account for the all of the present-day local abundances of ${ }^{6} \mathrm{Li},{ }^{9} \mathrm{Be}$, and ${ }^{10} \mathrm{~B}$ [1]. Other sources of ${ }^{7} \mathrm{Li}$ and ${ }^{11} \mathrm{~B}$ are required in addition to spallogenic mechanisms, leading to the suggestion that a significant component of the observable ${ }^{7} \mathrm{Li}$ in the ISM is a product of Big Bang nucleosynthesis [3]. Calculations by Woosley and Weaver suggest another small contribution to ${ }^{7} \mathrm{Li}$ and ${ }^{11} \mathrm{~B}$ galactic abundances from neutrino-driven spallation of ${ }^{12} \mathrm{C}$ within Type II supernovae[4].

The observed elemental ratios $\mathrm{Be} / \mathrm{H}, \mathrm{B} / \mathrm{H}$, and $\mathrm{Fe} / \mathrm{H}$ in low-metallicity halo stars formed in the early Galaxy tell a different story, indicating an overabundance of $\mathrm{LiBeB}$ in early epochs that cannot be accounted for by fragmentation of cosmic-ray CNO (e.g.,[5]). This interpretation assumes that the average ISM in any epoch serves as a source of material both for star formation and for cosmic rays in that epoch [6], and that these contribute $\mathrm{LiBeB}$ and other fragmentation products to the ISM at a somewhat later time. To explain both GCR spallogenic calculations and halo star abundances, possible solutions are that $\mathrm{LiBeB}$ species are created predominantly via fragmentation in the ISM of low-energy CO nuclei from SN II and Wolf-Rayet stars [7], or that cosmic rays are accelerated out of the metal-enriched supernova ejecta in superbubbles [8].

To address the origin of the $\mathrm{LiBeB}$ species, a precise calculation of the contribution from GCR fragmentation is needed. Our group has been using a simple cosmicray transport model to simulate cosmic-ray propagation in the galaxy for $4 \leq \mathrm{Z} \leq 28$, based upon the formalism of Meneguzzi, Audouze, and Reeves [9], and we have recently begun work on improving this model to pre-

CP598, Solar and Galactic Composition, edited by R. F. Wimmer-Schweingruber

(C) 2001 American Institute of Physics 0-7354-0042-3/01/\$18.00 
dict present epoch LiBeB GCR isotopic abundances and spectra observed at Earth. Because of their importance in any GCR transport model, we have re-examined fragmentation cross-section data in the scientific literature appearing both before and after the work of Read and Viola[10]. To test our model predictions, we used new abundance measurements made by the Cosmic Ray Isotope Spectrometer (CRIS) during the past three years [11]. The precision of the dataset from CRIS, $\sim 14 \%$ for statistical and systematic uncertainties in the $\mathrm{LiBeB}$ abundances, is high enough that cross-section uncertainties in the model must be considered. We have calculated the uncertainties that cross sections contribute to our model predictions. To determine in future studies whether we find an inconsistency between $\mathrm{LiBeB}$ halo star and spallogenic abundances, we wish to extend our predictions of the $\mathrm{LiBeB}$ production rate in the GCRs to lower energy. This includes using GCR H and He spectra from other experiments to predict $\mathrm{LiBeB}$ production rates from fragmentation of CNO atoms in the ISM and from $\alpha-\alpha$ fusion.

\section{PROPAGATION MODEL}

A steady-state, leaky-box model (e.g., [12]) was used for alculating the post-propagation GCR abundances observed by CRIS. Previously, abundance predictions from this model for cosmic-ray clock species led to the determination of the cosmic-ray confinement time [13]. A thorough review of the model input parameters (e.g., ionization fraction, ISM composition) was conducted by Yanasak et al. [13] to insure consistency with current literature. These parameters are similar to Davis, et al. [15], with a slight adjustment in the average value of the mean GCR pathlength through the ISM before escape to account for the lower ionization fraction used in this study. Abundance predictions for $\mathrm{Z}>4$ are virtually identical for both models.

With the availability of precise cosmic-ray data from CRIS, uncertainties in the fragmentation cross sections have become a dominant limitation to the study of rare cosmic-ray species that are generated predominantly via spallation [13]. A re-examination of cross-section estimates and their uncertainties was undertaken for reactions involving ${ }^{7} \mathrm{Be}, \mathrm{Li}$ isotopes, and products decaying to $\mathrm{LiBeB}$ species (e.g., ${ }^{6} \mathrm{He},{ }^{10,11} \mathrm{C}$ ), to include recent cross-section measurements. Partial and total fragmentation cross sections for nuclei of mass $A=9-56$ were previously updated in Yanasak et al. [13]. The "excitation functions" of Read and Viola [10], based on previous cross-section measurements, provide an estimate of isobaric production cross sections as a function of energy, and these are useful for predicting thermal LiBeB abun- dances in the ISM. However, species such as ${ }^{7} \mathrm{Be}$ and ${ }^{10} \mathrm{Be}$ which ultimately decay to ${ }^{7} \mathrm{Li}$ and ${ }^{10} \mathrm{~B}$ are significantly abundant in the GCRs, and their partial production cross sections are necessary for our model. Michel et al. [16] and Sisterson et al. [17] have precisely surveyed the partial Be production cross sections for fragmentation of $\mathrm{CNO}$ on hydrogen over a range of energies $E \sim 10-400 \mathrm{MeV} /$ nucleon. Higher energy crosssection measurements (E 365-600 MeV/nucleon, from $[18,19])$ for $\mathrm{p}+\mathrm{CNO} \rightarrow \mathrm{LiBeB}$ reactions have also been made since the compilation of Read and Viola. In addition, measurements for $\mathrm{He}+\mathrm{CNO} \rightarrow \mathrm{LiBeB}$ reactions are now available $[18,20])$.

In this study, three different methods for evaluating partial cross sections were used. For some reactions, enough cross-section data exist to define a function which can be interpolated at a particular energy to determine a cross-section value (Method 1). For other reactions which have a small number of measurements, the excitation functions of Read and Viola provided an energy dependence, and these were normalized where necessary for agreement with available cross-section data (Method 2). Finally, in cases where the parent nucleus is not CNO, the energy dependence of Silberberg, Tsao, and Barghouty [14] was used (Method 3a), and the cross sections for He-induced fragmentation in these cases were scaled using the parameterization of Hirzebruch, Winkel, and Heinrich [21]. For collisions with parent species $A>16$, the Silberberg et al. [14] energy dependence was also normalized to cross-section data where it exists (Method 3b). As a check, the isobaric cross-section data compiled in Read and Viola were compared to the sum of partial cross sections from the above methods, for reactions involving $\mathrm{CNO}$ parent nuclei.

Table 1 compares the contribution to $\mathrm{LiBeB}$ abundances from each of the three methods for calculating partial cross sections, typical uncertainties associated with cross-section measurements using each method, and the uncertainty contribution to the $\mathrm{LiBeB}$ abundances. The \% secondary contributions to $\mathrm{LiBeB}$ species were determined by using our model to compute abundances after setting the cross sections for other methods equal to zero. Tertiary reactions, where $\mathrm{B}$ nuclei fragment to $\mathrm{Li}$ and $\mathrm{Be}$, were not included in the calculation of the $\%$ contributions in Table 1 , and their importance will be discussed later. Using the average cross-section uncertainties for each method, the amount of uncertainty in the total predicted $\mathrm{LiBeB}$ abundances was calculated assuming a steady-state solution of the leaky-box model and following the formalism of Wiedenbeck [22] adapted for use with secondary GCR species. The average crosssection uncertainties for each method were derived differently. For Methods 2 and $3 b$ where few cross-section measurements exist, the average uncertainty was taken 
TABLE 1. Cross-section contributions to uncertainties in predicted LiBeB abundances

\begin{tabular}{|c|c|c|c|c|}
\hline $\begin{array}{l}\text { Cross-sectional } \\
\text { energy dependence } \\
\text { function }\end{array}$ & $\begin{array}{l}\text { Examples of } \\
\text { reactions }\end{array}$ & $\begin{array}{c}(\mathrm{Li}, \mathrm{Be}, \mathrm{B}) \% \\
\text { secondary } \\
\text { contributions * }\end{array}$ & $\begin{array}{c}\text { Typical } \\
\text { cross section } \\
\text { uncertainty }^{\dagger}\end{array}$ & $\begin{array}{c}\% \text { Uncertainty } \\
\text { in total predicted } \\
(\mathrm{Li}, \mathrm{Be}, \mathrm{B}) \text { abundance }\end{array}$ \\
\hline Interpolated function & $\begin{array}{c}\mathrm{p}+\mathrm{CNO} \rightarrow \mathrm{Be} \\
\mathrm{p}+\left({ }^{12} \mathrm{C},{ }^{16} \mathrm{O}\right) \rightarrow \mathrm{Li}, \mathrm{B}\end{array}$ & $(62.5,78.5,61.1) \%$ & $6 \%$ (p:5\%, He:6\%) & $(2.6,2.7,1.9) \%$ \\
\hline Read and Viola & $\begin{array}{c}\mathrm{p}+{ }^{14} \mathrm{~N} \rightarrow \mathrm{Li}, \mathrm{B} \\
\mathrm{He}+\left({ }^{12} \mathrm{C},{ }^{16} \mathrm{O}\right) \rightarrow \mathrm{Li}\end{array}$ & $(21.0,2.8,26.1) \%$ & $13 \%$ (p:17\%, He:10\%) & $(1.5,0.4,2.4) \%$ \\
\hline $\begin{array}{l}\text { Silberberg et al. } \\
\text { (normalized to data) }\end{array}$ & $\mathrm{p}+{ }^{22} \mathrm{Ne} \rightarrow \mathrm{B}$ & $(0.8,4.6,3.9) \%$ & $14 \% *^{* *}$ & $(0.0,<0.1,<0.1) \%$ \\
\hline (no data) & $(\mathrm{He}, \mathrm{p})+(\mathrm{A}>16) \rightarrow \mathrm{LiBeB}$ & $(15.7,14.1,8.9) \%$ & $\begin{array}{c}20 \%[14] \\
(\mathrm{p}: 20 \%, \mathrm{He}: 40 \%)\end{array}$ & $(0.3,0.3,0.1) \%^{\ddagger}$ \\
\hline
\end{tabular}

* Excluding tertiary reactions (e.g., $\mathrm{p}+\mathrm{B} \rightarrow \mathrm{Li}$ ).

$\dagger$ For an individual reaction, between $\mathrm{E}=100-10000 \mathrm{MeV} /$ nucleon

** No He cross-section measurements in this class

$\ddagger$ Corresponds to $40 \%$ uncertainty in the He cross sections. as the reduced standard deviation $\sigma=1 / \sqrt{\sum_{i}\left(1 / \sigma_{i}^{2}\right)}$, where $\sigma_{i}$ is an individual measurement uncertainty. For Method 1, an average percent deviation off the function weighted by measurement uncertainties was calculated as well as a reduced $\chi^{2}$ comparing the measurements with the average. For most reactions, $\chi^{2}$ was smaller than one, and the uncertainty was taken as the reduced standard deviation. For a few reactions, fluctuations not represented by the measurement uncertainties result in a large $\chi^{2}$, and in these cases, the actual reduced standard deviation was estimated by making $\sigma^{2}=\chi^{2} / \sqrt{\sum_{i}\left(1 / \sigma_{i}^{2}\right)}$

To estimate the uncertainty for reactions without any measurements (Method 3a), we compared the formulae of Silberberg et al. [14] for well-determined reactions to cross-section measurements. Silberberg et al. [14] estimate a general uncertainty in their formulae of $20 \%$, and we found a similar standard deviation in the measurement distribution around their formulae. However, for reactions induced by ISM helium, the standard deviation is significantly larger (from $\sim 0.4-2.0$ ). The reason for this may be that the scaling of Hirzebruch et al. [21] was determined for $\mathrm{E}_{\mathrm{ISM}} \geq 700 \mathrm{MeV} /$ nucleon and may break down at lower energies. The function of Tsao et al. [23] that scales ISM hydrogen reactions to helium will be compared in the future to Hirzebruch et al. [21] to determine if there is better agreement.

As shown in Table 1, the efforts of many previous cross-section experiments have collectively resulted in measurements for reactions that contribute almost $85 \%$ to the total $\mathrm{LiBeB}$ GCR abundances. In addition to these reactions, the contribution from fragmentation of $B$ isotopes to ${ }^{6} \mathrm{He}$ (which decays to ${ }^{6} \mathrm{Li}$ ), $\mathrm{Li}$, and $\mathrm{Be}$ may not be negligible in all cases because of the large relative abundance of $\mathrm{B}$ to $\mathrm{Li}$ and $\mathrm{Be}$. Cross-section measurements are available for the $\mathrm{p}+\mathrm{B} \rightarrow \mathrm{Be}$ reactions, and their uncertainties contribute $1 \%$ to the total $\mathrm{Be}$ abundance uncertainty. Unfortunately, we are not aware of the existence of high energy measurements of $\mathrm{p}+\mathrm{B} \rightarrow \mathrm{Li}$. Using the formulae of Silberberg et al. [14], the fragmentation of B boosts the abundance of Li from secondary reactions by an additional $\sim 12 \%$. Although the uncertainty for this reaction is unknown, a $20 \%$ cross-section uncertainty [14] results in a maximum $3 \% \mathrm{Li}$ abundance uncertainty. Adding the abundance uncertainties in Table 1 in quadrature, and adding $1 \%$ to $\mathrm{Be}$ and $3 \%$ to $\mathrm{Li}$ for uncertainties from tertiary reactions, we find that cross-section uncertainties affect the total predicted $\mathrm{LiBeB}$ elemental abundances in any model by $\sim 4.3 \%, 2.9 \%$, and $2.5 \%$ for $\mathrm{Li}, \mathrm{Be}$, and $\mathrm{B}$, respectively. It is important to note that all crosssection experimental measurements were assumed to be uncorrelated in this discussion. Multiple measurements for particular reactions exist that were made by individual experiments, and these data may suffer from correlated shifts in the cross-section normalization, resulting in somewhat larger abundance uncertainties than those derived above. However, the number of cross-section experiments surveyed by this study $(>70)$ that measure dominant reactions producing $\mathrm{LiBeB}$ is greater than the number of those reactions $(\lesssim 24)$, so uncertainty correlations are suppressed in general.

The total fragmentation cross sections were also updated in this study, although the long interaction length for $\mathrm{LiBeB}$ in hydrogen compared to the mean transport pathlength through the ISM makes uncertainties for these less important. The energy dependence of total fragmentation formulae from Letaw, Silberberg, and Tsao [25] 

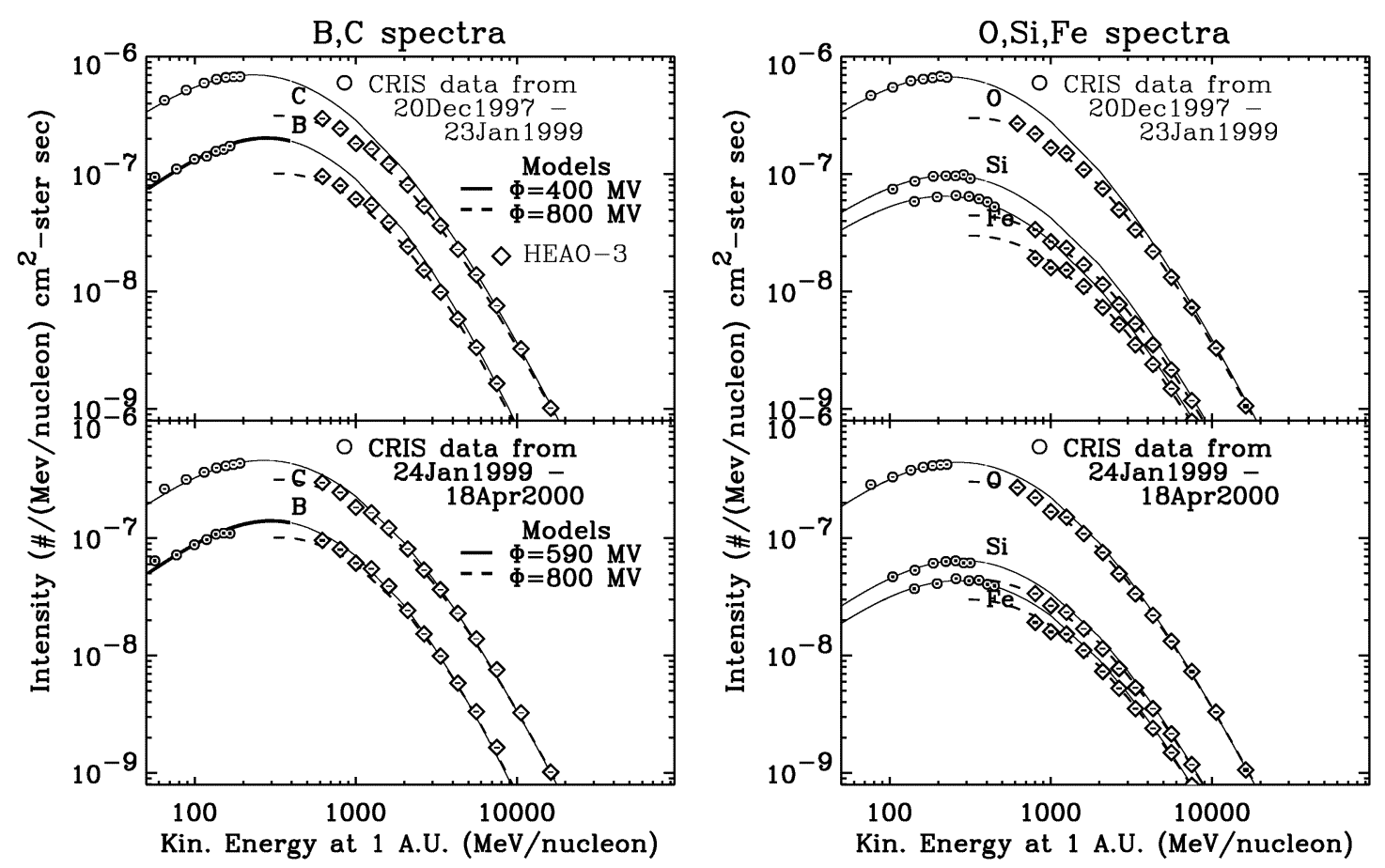

FIGURE 1. Flux measurements from CRIS for B and abundant primary GCR species. Data from the HEAO-3 spacecraft are shown for comparison [24]. Also shown are predictions for CRIS spectra from our model (solid line), uncertainties for B predictions (thick line below $400 \mathrm{MeV} /$ nucleon), and model predictions at a higher level of modulation $\phi=800 \mathrm{MV}$ comparable to HEAO-3 data (dashed line).

is consistent with $\mathrm{LiBeB}$ cross-section data at GCR energies in the ISM probed by CRIS ( $\mathrm{E}_{\mathrm{ISM}} \sim 200-500$ $\mathrm{MeV} /$ nucleon), and at all energies for $\mathrm{Z} \geq 8$. However, at lower energies relevant to galactic evolution studies, the formulae of Tripathi, Cucinotta, and Wilson [26] are in better agreement with $\mathrm{LiBeB}$ data. A total fragmentation cross-section energy dependence is chosen which matches Letaw et al. at the high energies and Tripathi et al. at lower energies.

Our steady-state model implicitly uses an exponential GCR pathlength distribution. The mean ISM pathlength was adjusted to match four secondary-to-primary ratios from CRIS $(\mathrm{B} / \mathrm{C}, \mathrm{F} / \mathrm{Ne}, \mathrm{P} / \mathrm{S}$, and $(\mathrm{Sc}+\mathrm{Ti}+\mathrm{V}) / \mathrm{Fe})$, using HEAO-3 data at higher energies as a measure of consistency [24]. Solar modulation of the GCR spectra was simulated using the spherically-symmetric model described by Fisk [27]. Levels of modulation were determined via choosing a source spectrum and matching post-propagation spectral shapes in our model to HEAO3 and CRIS data [15].

\section{DISCUSSION}

Predictions for the absolute spectrum of $\mathrm{B}$ as well as other GCR dominant nuclei $\mathrm{C}, \mathrm{O}, \mathrm{Si}$, and $\mathrm{Fe}$ are shown in Figure 1. The thicker curve shown for the predicted B spectrum at $\mathrm{E} \lesssim 200 \mathrm{MeV} /$ nucleon indicates one standard deviation of uncertainty from cross sections. Data from CRIS and HEAO-3[24] match both the absolute intensities and energy dependences of the predicted spectra well for $\mathrm{B}, \mathrm{C}, \mathrm{O}, \mathrm{Si}$, and $\mathrm{Fe}$ during both time periods chosen. Statistical uncertainties for the CRIS are small compared with the size of the plotted circles, and an additional $\sim 10 \%$ uncertainty in the instrument systematics should also be added in quadrature [11].

Figure 2 shows comparisons between isotopic ratios from model predictions and data from the first period in Figure 1. Small corrections $(\leq 10 \%)$ have been made to the data to account for differences in average measurement energy of the data for each species. Uncertainties for CRIS in this figure account for both statistical and systematic uncertainties. The hatched regions shown in Figure 2 are one standard deviation of uncertainty in the model prediction from cross sections, somewhat larger than those shown in Table 1 for elemental species because the uncertainties for individual isotopes are considered. The average value of the data and the model generally agree, although model uncertainties prevent a useful comparison to the energy dependences of the data. In particular, the ${ }^{7} \mathrm{Be} /{ }^{9} \mathrm{Be}$ ratio shows slightly more energy dependence than what is expected from the model. However, the uncertainty contribution from the 


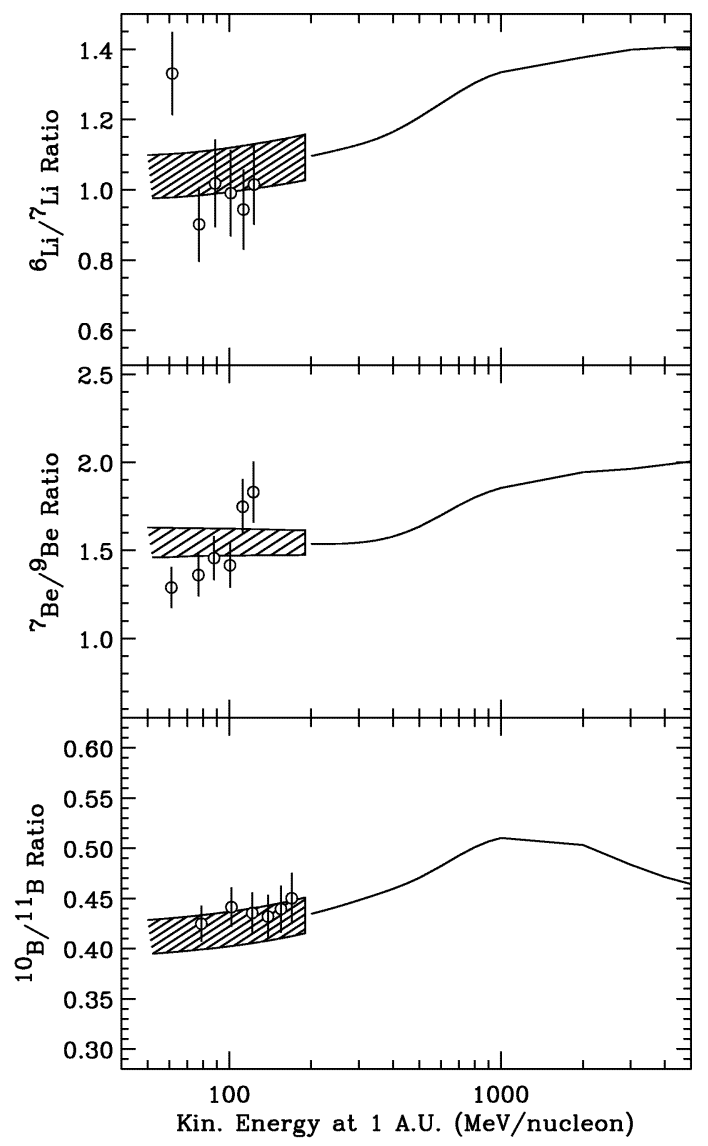

FIGURE 2. Relative isotopic abundances from CRIS (open circles) and model predictions (solid line). The hatched region indicates one standard deviation from cross-section uncertainties. Note that the $y$-axis scale for each ratio is different and has an offset.

$(\mathrm{p}, \mathrm{He})+\mathrm{B} \rightarrow \mathrm{Be}, \mathrm{Li}$ reactions is not included in the predicted uncertainties shown in Figure 2, which should improve the agreement.

We are achieving good agreement between CRIS data and our model for Be and B species, and our model predictions compare favorably with other computations of the GCR spallogenic contribution to $\mathrm{LiBeB}$ abundances. Propagation parameters from the model of GarciaMunoz et al. [29] are used by a number of studies (e.g., [5]), which give comparable results to our model within the uncertainty of $\pm 30 \%$ suggested by the authors for their GCR mean escape pathlength. The ratios of stable secondary isotopic species with the exception of ${ }^{10} \mathrm{~B}$, which has a small contribution from the decay of ${ }^{10} \mathrm{Be}$, should not be affected by differences in the choice of mean pathlength. Differences in the $\mathrm{Li} / \mathrm{C}$ and $\mathrm{Be} / \mathrm{C}$ ratios at 1 A.U. predicted by both models are $\lesssim 10 \%$ at $50 \mathrm{MeV} /$ nucleon, increasing to $20 \%$ at 1000 $\mathrm{MeV} /$ nucleon. More importantly, the mean pathlength from [29] is not independent of the choices for produc-

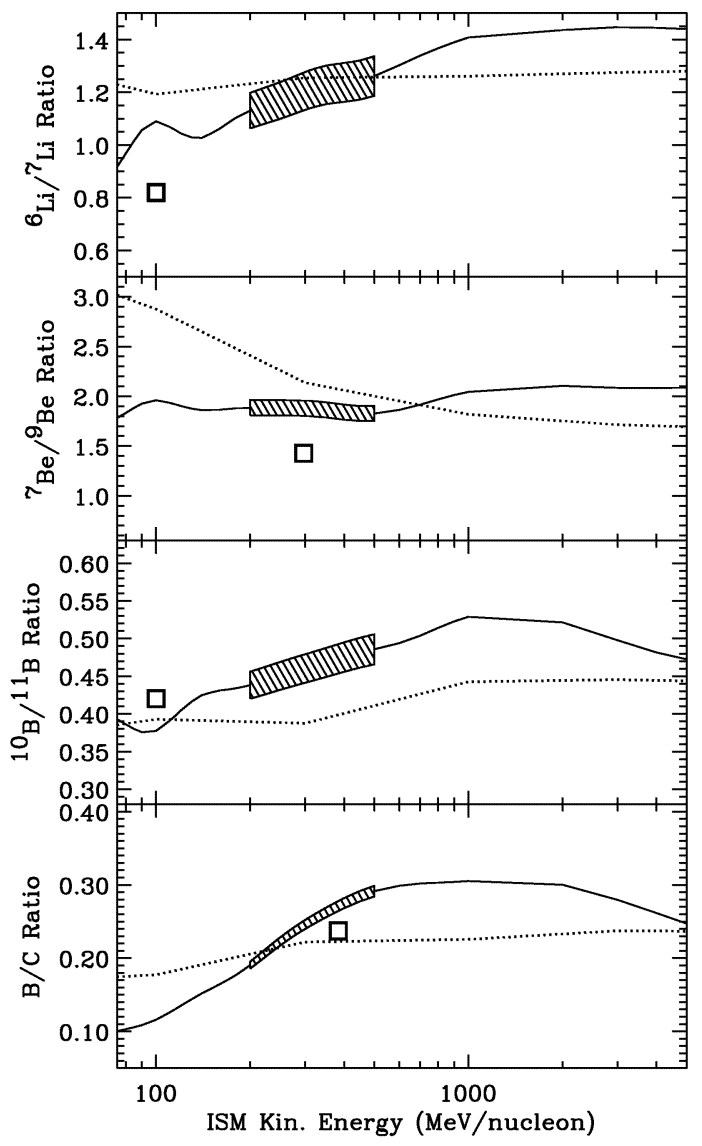

FIGURE 3. Model predictions in the ISM from this study (solid line). The size of the hatched region represents one standard deviation of model uncertainty (see Figure 2). The dotted line is a predictions from Meneguzzi et al. [9], and the squares are predictions from a model by Lukasiak, McDonald, and Webber [28].

tion and fragmentation cross sections presented in the appendix of that study. One must take care that any study incorporating new cross-section measurements and using a previously-reported GCR mean pathlength is able to predict adequately GCR abundance data for species produced via fragmentation.

Figure 3 shows predictions from our model in the ISM for several elemental and isotopic abundance ratios as solid line and a hatched band, with the width of the band representing the typical model uncertainty from cross sections. Also shown are GCR abundance predictions from Meneguzzi et al. [9] (dotted line) and from a Monte Carlo diffusion model of Lukasiak et al. [28] (boxes). The energy assignment for the ${ }^{7} \mathrm{Be} /{ }^{9} \mathrm{Be}$ and $\mathrm{B} / \mathrm{C}$ ratios from Lukasiak et al. [28] is somewhat uncertain because these data were measured within the solar system and extrapolated to the ISM. Agreement between the latter study and our model is good within uncertainties, with the possible exception of ${ }^{7} \mathrm{Be} /{ }^{9} \mathrm{Be}$. One possible ex- 
planation for the lower abundance of ${ }^{7} \mathrm{Be}$ predicted by Lukasiak et al. [28] is that the cross sections used in each study are different.

Unlike the work of Garcia-Munoz et al. [29] and Lukasiak et al. [28], the early model of Meneguzzi et al. [9] assumes a mean pathlength that is indepenent of energy, and the effect of this choice appears as a generally flat secondary-to-primary ratio $\mathrm{B} / \mathrm{C}$. At energies probed by CRIS, the agreement between our model and that of Meneguzzi et al. is satisfactory or shows a difference from a lack of measured cross sections at the time of the earlier study (e.g., ${ }^{10} \mathrm{~B} /{ }^{11} \mathrm{~B}$ ). Predictions by both models for the $\mathrm{B} / \mathrm{C}$ ratio at lower energies shows a significant difference $(\sim 1.8 \mathrm{x})$. Using an energy-dependent mean pathlength will effectively decrease the amount of $\mathrm{LiBeB}$ production from GCR CNO fragmentation at low energies relative to higher energies. Because the lower energy particles will thermalize more quickly than those with higher energies, the Meneguzzi et al. model should predict more production of $\mathrm{LiBeB}$ by GCR CNO parents with $\mathrm{E}_{\mathrm{ISM}}>100 \mathrm{MeV} /$ nucleon than our model.

\section{CONCLUSIONS}

We have surveyed model parameters at GCR energies probed by CRIS (E $\mathrm{E}_{\mathrm{ISM}} \sim 200-500 \mathrm{MeV} /$ nucleon). Our model gives a satisfactory prediction for GCR primary and secondary species with $Z \geq 4$, and it shows good agreement for relative isotopic abundances. In some cases (notably $(\mathrm{p}, \mathrm{He})+\mathrm{B} \rightarrow \mathrm{Li}$ ), a lack of cross-section measurements limits our understanding of the model inputs. However, with additional measurements made since Read and Viola [10], uncertainties for the cross sections for some reactions have improved, and we have calculated estimates of the magnitude of our uncertainty. Comparisons with previous models show general agreement in the energy range relevant to CRIS data. Future work will include turning our model around to predict the amount of $\mathrm{LiBeB}$ produced via inelastic, GCR $\mathrm{p}$ and $\mathrm{He}$ interactions with CNO in the ISM, and we will also investigate the low-energy contribution from $\alpha-\alpha$ fusion to ${ }^{6,7} \mathrm{Li}$.

\section{ACKNOWLEDGMENTS}

This research was supported by NASA at the California Institute of Technology, (grant NAG5-6912), the Jet Propulsion Laboratory, the NASA/Goddard Space Flight Center, and Washington University.

\section{REFERENCES}

1. Reeves, H., Rev. Mod. Phys., 66, 193 (1994).

2. Reeves, H., Fowler, W. A., and Hoyle, F., Nature Phys. Sci., 226, 727 (1970).

3. King, C. H., Austin, S. M., Rossner, H. H., and Chien, W. S., Phys. Rev. C, 16, 1712 (1977).

4. Woosley, S. E., and Weaver, T. A., Ap. J. Supp., 101, 181 (1995).

5. Lemoine, M., Vangioni-Flam, E., and Casse, M., Ap. J., 499, 735 (1998).

6. Vangioni-Flam, E., Casse, M., Audouze, J., and Oberto, Y., Ap. J., 364, 568 (1990).

7. Casse, M., Lehoucq, R., and Vangioni-Flam, E., Nature, 373, 318 (1995).

8. Higdon, H. C., Lingenfelter, R. E., and Ramaty, R., Proc. 26th Int. Cosmic-Ray Conf. (Salt Lake City), 4, 144 (1999).

9. Meneguzzi, M., Audouze, J., and Reeves, H., Astron. Astrophys., 15, 337 (1971).

10. Read, S. M., and Viola, V. E., Atomic Data Nucl. Data Tables, 31, 359 (1984).

11. de Nolfo, G. A., et al., this conference (2001).

12. Leske, R. A., Ap. J., 405, 567 (1993).

13. Yanasak, N. E., et al., Ap. J., submitted (2001).

14. Silberberg, R., Tsao, C. H., and Barghouty, A. F., Ap. J., 501, 911 (1998).

15. Davis, A. J., et al., "On the Low Energy Decrease in Galactic Cosmic Ray Secondary/Primary Ratios", in Acceleration and Transport of Energetic Particles Observed in the Heliosphere: ACE 2000 Symposium, edited by R. Mewaldt et al., AIP Conference Proceedings 528, American Institute of Physics, New York, 2000, p. 421.

16. Michel, R., et al., Nucl. Instr. and Meth. B, 103, 183 (1995).

17. Sisterson, J. M., et al., Nucl. Instr. and Meth. B, 123, 324 (1997).

18. Webber, W. R., Kish, J. C., and Schrier, D. A., Phys. Rev. $C, 41,547$ (1990).

19. Webber, W. R., et al., Ap. J., 508, 949 (1998).

20. Mercer, D. J., Austin, S. M., and Glagola, B. G., Phys. Rev. C, 55, 946 (1997).

21. Hirzebruch, S. E., Winkel, E., and Heinrich, W., Proc. 23rd Int. Cosmic-Ray Conf. (Calgary), 2, 175 (1993).

22. Wiedenbeck, M. E., "The Effect of Cross-Section Uncertainties on the Derivation of Source Abundances From Cosmic-Ray Composition Observations", in Composition and Origin of Cosmic Rays, edited by M. M. Shapiro, D. Reidel, Dordrecht, 1983, p. 343.

23. Tsao, C. H., Silberberg, R., and Barghouty, A. F., Ap. J., 501, 920 (1998).

24. Englemann, J. J., et al., Astron. Astrophys., 233, 96 (1990).

25. Letaw, J. R., Silberberg, R., and Tsao, C. H., Ap. J. Suppl., 51, 271 (1983).

26. Tripathi, R. K., Cucinotta, F. A., and Wilson, J. W., NASA Technical Report TP-1999-209726 (1999).

27. Fisk, L. A., JGR, 76, 221 (1971).

28. Lukasiak, A., McDonald, F. B., and Webber, W. R., Proc. 26rd Int. Cosmic-Ray Conf. (Salt Lake City), 3, 41 (1999).

29. Garcia-Munoz, M., Simpson, J. A., Guzik, T. G., Wefel, J. P., and Margolis, S. H., Ap. J. Suppl., 64, 269 (1987). 